\title{
"Voxelization" in 3D Searching- A Study
}

\author{
Tanuja Subba \\ M.Tech in CSE \\ Sikkim Manipal Inst. of \\ Technology \\ Majhitar, East Sikkim
}

\author{
Ashis Pradhan \\ Assistant Professor in CSE \\ Sikkim Manipal Inst. of \\ Technology \\ Majhitar, East Sikkim
}

\author{
Prerna Rai \\ Lecturer in Computer Science \\ Centre for Computer \& Comm. \\ Technology, South Sikkim
}

\begin{abstract}
Recycling of engineering design is increasing exponentially in order to reduce the conceptual designing phase time due to rapid increase in technology. This leads to increase in frequent retrieval of the $3 \mathrm{D}$ models through web searching mechanism. So, it becomes a necessary challenge to design a 3D search engine which can search 3D models accurately as well as efficiently. The 3D searching is a technique developed to help the web users for extracting 3D models like complex engineering parts, various purpose graphical models, etc. The search engine takes input in the form of queries like text, 2D sketches, 3D sketches, 3Dmodel, 2Dmodel etc. When input queries is matched with the existing model in the database or similar model that are present in the database, the best multiple result is provided to user. This paper describes the basics of query by sketch which takes input by sketching manually with the aid of user interface. This input is normalized and further processed by Voxelization, Skeltonization and create Skeletal graph which is then mapped to database to extract best possible match. However, the main focus of this paper is to discuss the process of Voxelization method in very simple steps, as it plays a very important aspect in 3D searching.
\end{abstract}

\section{Keywords}

3D models, Text keywords, 2D image, 3D image

\section{INTRODUCTION}

Designer spends most of their time searching appropriate result and it is rated as frustrating part for engineer. Engineering design and manufacturing has progressed extensively from $2 \mathrm{D}$ to $3 \mathrm{D}$ in the last decade. As a result, a number of new methodologies for 3D model have been created, leading to development of 3D search engine. 3D search engine is capable of retrieving similar model that a user wants this search engine is necessary in today's world as the number of use of 3D model has increased tremendously. One of the processes of searching the model is through search by sketch where process like Voxelization, Skeletonization and Skeletal graph takes place Voxelization is the method to convert 2D images, 3D images into set of voxel. Skeletonization is a process of extracting a skeleton from 3D image (voxel model) and the skeleton obtained is converted to hierarchical skeletal graph to store in database.

The paper describes the basics of query by sketch which takes input by sketching manually with the aid of user interface. This input is normalized and further processed by Voxelization, Skeltonization and create Skeletal graph which is then mapped to database to extract best possible match.
However, the main focus of this paper is to discuss the process of Voxelization method in very simple steps, as it plays a very important aspect in 3D searching.

\section{RELATED WORK}

A number of 3D model and searching techniques have developed so far as because the use these have increased and played an effective role in designers and engineers. Some of them can be classified as follows:

a) Invariant/Descriptor based: These method use invariants or descriptor of the 3D shape such as volume, surface area, aspect ratio, higher order moments or moment invariants as signatures ${ }^{[1]}$.

b) Harmonics based: These approaches use a set of harmonic functions of a shape as its signature. Spherical or Fourier functions are usually used to decompose a discrete 3D model into an approximate sum of its (first $n$ ) harmonic components ${ }^{[2]}$.

c) 3D Object Recognition based: some 3D object recognition approaches that have been used for 3D shape searching are Aspect Graphs ${ }^{[3]}$, Spin Images ${ }^{[4]}$, and Geometric Hashing ${ }^{[5]}$

d) Graph based: Graph based approaches have employed subgraph ${ }^{[6]}$, matching eigen values of a model signature graph (MSG) constructed from B-Rep graph ${ }^{[7]}$. A multiresolution Reeb graph that captures the topology of a part was proposed in ${ }^{[8]}$.

e) Feature Recognition Based: Ramesh et al. ${ }^{[9]}$ decompose a part into cells which are further processed to identify machining features and their spatial relationships to calculate similarity between parts. Ascher et al. [10] simplify parts into maximal feature subgraphs (MFSG) which are matched to obtain a similarity measure.

\section{SYSTEM OVERVIEW OF MODEL}

The organization of a $3 \mathrm{D}$ model is shown in the figure 1 . The major components of the model are: crawler, indexing, query and matching.

i. Crawling: A database of 3D models is built by crawling the web. A crawler incorporates a measure of 3D model "quality" into its page rank.

ii. Indexing: an index is computed to retrieve 3D models efficiently based on text and shape queries.

iii. Querying: allows a user to search for 3D models. It will support query methods based on text keywords, 2D sketching, 3D sketching, model matching, and iterative refinement.

iv. Matching: For any user query index is used to return the models that are best matched with the query. 


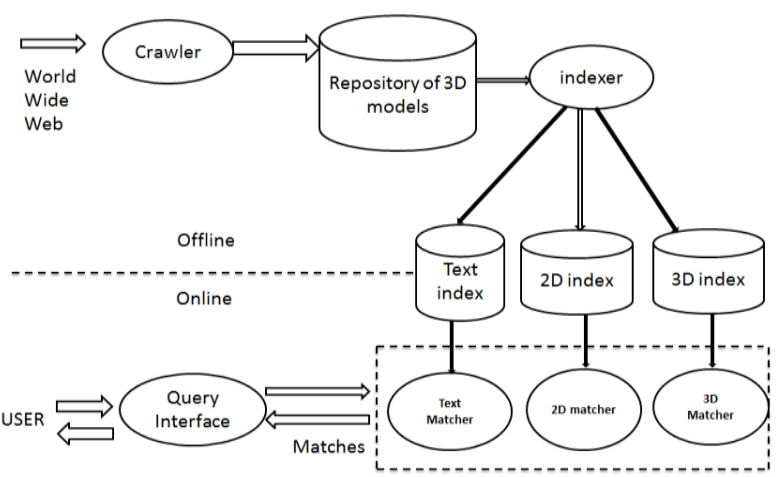

Figure 1: System overview ${ }^{[11]}$

\section{SEARCH PROCESS}

The basic Client-Server-Database architecture is shown in figure 2 . The client provides interface to the user and displays the result. The user can search model through queries like text, 2D images, 3D images etc and Server side takes in the input from the Client and converts it into multiple representations (voxel, skeleton, feature vectors and skeletal graph), which are then stored into the Database. This paper focuses on the query by sketching where user can draw 2D model/3D model. This drawn image is then converted into voxel i.e. set of volmue cube with the process of voxelization and then transferred into skeletonization then to skeletal graph and the result is searched through database.

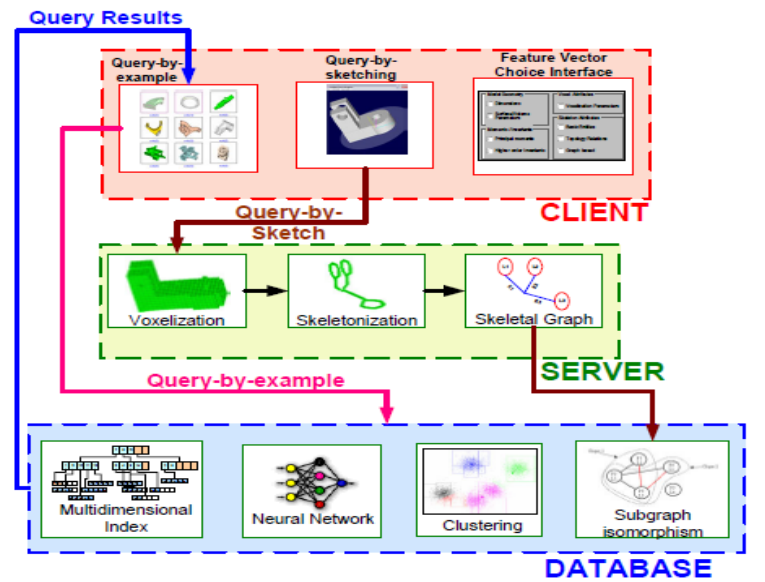

Figure 2: A different query process and its component ${ }^{[12]}$

\section{VOXELIZATION}

The 3D search system uses algorithm to convert the selected or drawn image into a mathematical model called the voxel model by a process of Voxelization. Voxelization is the process of approximating a continuous object by a set of voxel i.e. the data of an object is stored in a set of regular $3 \mathrm{D}$ grids. Voxelization is a 3D scan conversion and the difference between voxelization and 2D scan conversion is it decouples object specific issues from rendering (generating an image from $2 \mathrm{D}$ or $3 \mathrm{D}$ model by means of computer programs) and enables uniform representation of all objects by a simple primitive voxel that is it simplifies its architecture and leads to a more effective implementation by means of analytic formulas. Voxel generated can be binary or multi-valued. A voxel is the unit cubic volume centered at the integral grid point the voxel value is mapped on the set of $\{0,1\}$ voxel value with 1 represents "black" or "non-empty" voxel and 0 represents "white" or "empty" voxel. The voxel neighborhood relationship can be divided into three kinds 6-neighborhood, 2 voxels share a face; 18 -neighborhood, 2 voxels share a face or an edge; 26-Neighborhood, 2 voxels share a face, an edge or a vertex.

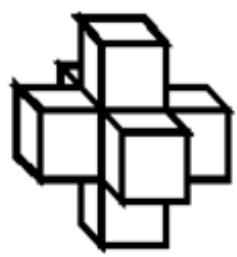

(i)

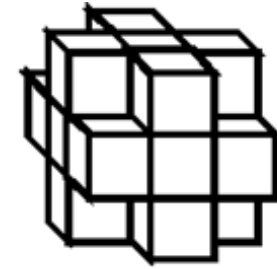

(ii)

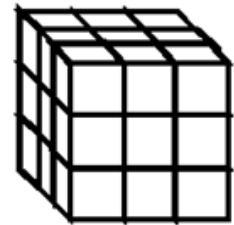

(iii)

Figure 3: $\mathrm{N}$-neighborhood diagram of the voxel $\mathrm{N} \in\{6(\mathrm{i})$, 18(ii), 26(iii)\}

The used voxelization methods are boundary representation (B-Rep) and constructive solid geometry (CSG). B-Rep defines parts in terms of vertices, edges and faces. CSG defines parts in terms of set of Boolean operations applied to primitive geometry.

The Voxelization Algorithm: After voxelization, a 3D volume model can be obtained. Because the feature extraction of 3D model retrieval only needs surface date, voxelization is actually the process to voxelize all the patches which compose the model surface. Since, the voxelization for polygons is common, the steps involved in such process are as follows:

Step1: Voxelizing Vertices: For each vertex, we define a bounding sphere of radius Rc as shown in Fig.3. All the voxels whose voxel center fall inside any one of the bounding spheres is equal to 1 .

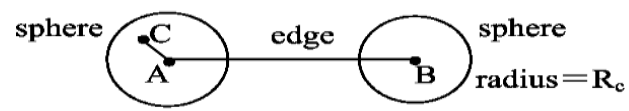

Figure 3: Voxelizing vertices ${ }^{[13]}$

Considering the case as shown in figure 3 , assume a point $\mathrm{A}$ $(\mathrm{x} 1, \mathrm{y} 1, \mathrm{z} 1)$, point $\mathrm{B}(\mathrm{x} 2, \mathrm{y} 2, \mathrm{z} 2)$ are two vertices of a triangular patches and point $\mathrm{C}(\mathrm{x} 0, \mathrm{y} 0, \mathrm{z} 0)$ is any voxel center, then the Euclidean distance between $\mathrm{C}$ to $\mathrm{A}$ is as:

$$
d=\sqrt{\left(x_{0}-x_{1}\right)^{2}+\left(y_{0}-y_{1}\right)^{2}+\left(z_{0}-z_{1}\right)^{2}}
$$

Step 2: Voxelizing Edges: For each edge, we define a regular bounding cylinder of radius $\mathrm{Rc}$ and length $\mathrm{L}$, where $\mathrm{L}$ is the length of the edge, as shown in Fig.4. All the voxels whose voxel centers fall inside any one of the bounding cylinders equal to 1 .

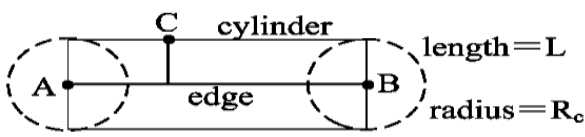

Figure 4: Voxelizing edges [13] 
Considering the case as shown in figure 4 , assume a point $\mathrm{A}$ $(\mathrm{x} 1, \mathrm{y} 1, \mathrm{z} 1)$, point $\mathrm{B}(\mathrm{x} 2, \mathrm{y} 2, \mathrm{z} 2)$ are two vertices of a triangular patches and point $\mathrm{C}(\mathrm{x} 0, \mathrm{y} 0, \mathrm{z} 0)$ is any voxel center. The distance from voxel center $\mathrm{C}$ to the edge $\mathrm{AB}$ is calculated as:

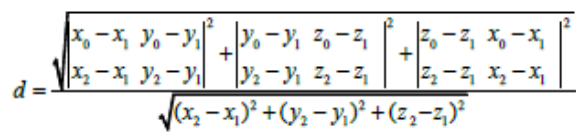

Step3: Voxelizing Surface: For each triangular patch S, we construct two parallel planes such that $\mathrm{S}$ lies between these planes and is parallel to both of them, and the distance from $S$ to $\mathrm{G}$ or $\mathrm{H}$ is $\mathrm{t}$, as shown in Fig. 5. All the voxels with their voxel center falling inside this triangular prism is equal to 1 .

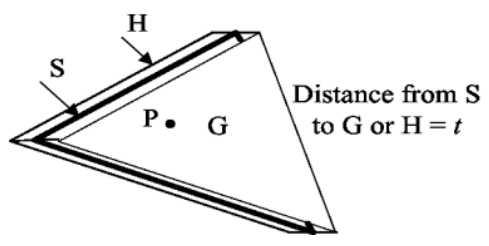

Figure 5: Voxelizing surface ${ }^{[13]}$

Considering the case as shown in figure 5 , assume surface $S$ be a triangular patch, where $(S=A x+B y+C z+D=0)$, point $\mathrm{P}(\mathrm{x} 0, \mathrm{y} 0, \mathrm{z} 0)$ is any voxel center, then the distance from voxel's center $\mathrm{C}$ to the triangular patch $\mathrm{S}$ is calculated as:

$$
d=\frac{\left|A x_{0}+B y_{0}+C z_{0}+D\right|}{\sqrt{A^{2}+B^{2}+C^{2}}}
$$

After Voxelization is done the model obtained is shown in fig 6 and it should satisfy separability and minimality. Separability and minimality is a topological property and hence, voxelization is a topological approach rather than geometrical.

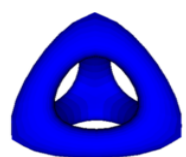

Voxelization
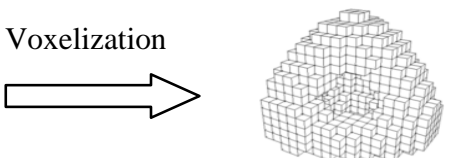

Figure 6: Voxel model

Separability states that voxelization must be thick to prevent any rays from penetrating the surface that is the surface should not contain any holes. Minimality ensures minimum nuber of voxel used to represent separability i.e. deleting a voxel should not affect the separability of a voxel model which gives an efficient representation.

\section{SKELETONIZATION}

Skeletonization is the process of extracting a skeleton from $3 \mathrm{D}$ voxel model. A skeleton is composed of skeletal points and skeletal entities (can be a line or a loop entity). It must represent:

i. Topological: A skeleton must retain the topology of the original object.

ii. Geometrical: A skeleton must be in the "middle" of the original object and must be invariant to translation, rotation and scaling.

Skeletonization is achieved by various algorithm like thinning, voronoi's diagram, distance transform based etc.

\section{SKELTAL GRAPH}

This graph is undirected graph consisting of vertices $(\mathrm{V})$ and edges (E). V represents skeletal line or loop and E represents connectivity between skeletal entities. This graph can be analyzed at different levels depending on information content.

The skeleton is converted into a skeletal graph made up of the following basic skeletal entities:

i. Skeletal abstraction has to be extracted from the skeleton.

ii. Identify those voxels which are end points and junction points.

iii. Point- the voxel situated at the ends of the segment and at the junction.

iv. Entities - Loops and segments (edges) which are further divided into straight line and curve.

Each edge in the skeleton translates into an independent geometric entity giving a shape to the model in physical space. Similarly, each loop represents a hole in the 3D model. For example, if a user wants to search for models with holes, it can be completed by searching for graphs with loops ${ }^{[12]}$.

\section{CONCLUSION}

3D search engine is most efficient system used for extracting 3D models for user. Querys like text, keyword, 2D image,3D sketch is given as input and the matched or appropriate output is given to the user.

The Voxelization method is used for extracting images in terms of volume pixel known as voxel followed by Skeletonization and Skeletal graph which looks for isomorphic structure in the database and returns the most matched graph to the user.

\section{REFERENCES}

[1] Cybenko, G., Bhasin, A., and Cohen, k.,(1997)," "Pattern Recognition of 3D CAD Objects", Smart Engineerinf System Design, Vol 1,pp.1-13.

[2] Kazhdan., M., Chazelle, B,., Dobkin, d., Finkelstein, A., and Funkhouser, T., (2002), "A Reflrctive Symmetry Descriptor", Proceedinfs of 7th European Conference on Computer Vision, Copenhagen, Denmark, pp. 642-656

[3] Cyr, C.M., and Kimia, B., (2001), “3D Object Recognition Using Shape Similarity Based Aspect Graph”, Proceedings of ICCV'01, pp.254-261

[4] Ruiz-Correa, S., Shapiro, L. and Meila, M.,(2000). "A New Signature Based Method For Efficient 3D Object Recognition", Proceedings of VPR'00, S

[5] Lamdam, Y. and Wolfson, H.J., (1988), "Geometric Hashing: A General And Efficient Model Based Recognition Scheme”, Proceedings of ICCV'88

[6] El-Mehalawi, M., and Miller, R., (2003), “ A database system of mechanical components based on geometric and topological similarity. Part I: representation and Part II: indexing, retrieval, matching, and similarity assessment", Computer Aided Design, Vol. 35, pp. 83-94

[7] Sun, T-L., Su, C-J., Mayer, R., and Wysk, R,. (1995), “ Shape Similatity Assesment Of Mechanical parts based on solid models" Proceedings of ASME DFM Conference, Boston, MA, pp. 953-962 
[8] Hilaga, M., Shinagawa, Y., Kohmura, T., and Kunni, T., (2001), "Topology matching for fully automatic similarity estimation of 3D shapes", Proceedings of SIGGRAPH'01, pp. 203-212

[9] Ramesh . M., Yip-Hoi, D., Dutta, D., (2001), "Feature Based Shape Similarity Measurement For Retrieval Of Mechanical Parts", Vol. 1, pp. 245-256

[10] Ascher, M., Marefat, M, and Fasse, E,(2001), "A methodology for automatic retrieval of similarly shaped machinable components", IEEE Interanational Conference on Systems, Man, and Cybernetics, Vol. 2, pp. $2840-2845$

[11] Pranav Agrawal, "3D Searching”, Global Journals inc.(USA) 0975-4172 and Print ISSN: 0975-4350

[12] Natraj Iyer, Kuiyang Lou, Subramaniam Jayanti ,et. al., "Early Results from 3DESS: A 3D Engineering Shape
Search System", School of Mechanical Engineering, Purdue University West Lafayette IN 47906, USA

[13] XuHaili, Wang Heng, Zhu Longbiao and HuaGuoran, “ Reading and voxelization of 3D models",School of Mechanical Engineering, Nantong University, Nantong, Jiangsu, China.

[14] Natraj Iyer, Yagnanarayanan Kalyanaraman, et.al., "A Reconfigurable 3D Engineering Shape Search System", Proceedings of ASME DETC' 03, 23rd Computers and Information in engineering (CIE) Conference, September 2-6, 2003, Chicago, Illinois.

[15] Suyu Hou, Karthik Ramani "Dynamic Query Interface For 3D Shape Search Proceedings of ASME IDETC/CIE 2004, Salt Lake City, UT, USA. 